\title{
The potential of nanomedicine to alter cancer stem cell dynamics: the impact of extracellular vesicles
}

\author{
Petra Gener ${ }^{1}$ (D), Patricia Gonzalez Callejo ${ }^{1,2}$, Joaquín Seras-Franzoso ${ }^{1}$, Fernanda Andrade ${ }^{1}$, \\ Diana Rafael ${ }^{1,2}$, Ibane Abasolo ${ }^{1,2}$ \& Simo Schwartz Jr*,1,2 \\ ${ }^{1}$ Drug Delivery \& Targeting Group, Molecular Biology \& Biochemistry Research Centre for Nanomedicine (CIBBIM-Nanomedicine), \\ Vall d'Hebron Institut de Recerca, Universitat Autònoma de Barcelona, 08035 Barcelona, Spain \\ ${ }^{2}$ Networking Research Centre for Bioengineering, Biomaterials \& Nanomedicine (CIBER-BBN), Instituto de Salud Carlos III, 28029 \\ Madrid, Spain \\ *Author for correspondence: simo.schwartz@vhir.org
}

The presence of highly resistant cancer stem cells (CSCs) within tumors as drivers of metastatic spread has been commonly accepted. Nonetheless, the likelihood of its dynamic phenotype has been strongly discussed. Importantly, intratumoral cell-to-cell communication seems to act as the main regulatory mechanism of CSC reversion. Today, new strategies for cancer treatment focusing into modulating tumor cell intercommunication and the possibility to modulate the composition of the tumor microenvironment are being explored. In this review, we summarize the literature describing the phenomenon of CSC reversion and the factors known to influence this phenotypic switch. Furthermore, we will discuss the possible role of nanomedicine toward altering this reversion, and to influence the tumor microenvironment composition and the metastatic spread of the disease.

First draft submitted: 6 March 2020; Accepted for publication: 5 October 2020; Published online: 16 November 2020

Keywords: cancer stem cells • dynamic phenotype • extracellular vesicles • nanomedicine

\section{Cancer stem cell models (the hierarchic model, stochastic model \& dynamical tumor initiation model)}

Remarkable similarities between embryonic development and tumor growth were acknowledged as early as the 19th century [1]. Later, in 1953, Dixon and Moore postulated for the first time that tumor cells possess characteristics similar to germinal cells and concluded that, in most cases, undifferentiated cells are the origin of tumor development [2]. However, the real breakthrough regarding the cancer stem cell (CSC) theory of tumor development came 40 years later from studies on the origin of acute myeloid leukemia [3]. Those studies demonstrated that only few rare cells (CSCs) were capable of initiating leukemia in mice. A strong resemblance of these cells with hematopoietic stem cells (HSCs) was also shown. They expressed typical HSC markers $\left(\operatorname{lin}^{-} \mathrm{CD} 34^{+} \mathrm{CD} 38^{-}\right)$and showed high capacity for self-renewal and differentiation. Notably, tumors that appeared after the in vivo transplantation of only few CSCs in mice were composed of a mixture of cells that was very similar to the one found in the initial tumor [3,4]. Since the first description of leukemic stem cells, CSCs have been identified in many types of cancers, including solid tumors [5-9]. Ever since, the CSC theory has been constantly modified based on growing knowledge, mostly disputing CSC characterization principles and the basis of tumor initiation [10-13].

The CSC model was originally referred to as a hierarchic model in which cancer cells originate from a precursor stem cell (CSC). This precursor cell divides either symmetrically, giving two identical CSCs or two differentiated cancer cells (DCCs), or asymmetrically, giving one CSC and one DCC [3]. Accordingly, the distinctive ability of these cell types regarding tumor initiation has been considered as an indispensable feature to distinguish CSCs from DCCs $[3,14,15]$. The presence of CSCs that fits into the hierarchic model has been demonstrated in some models of breast cancer where as few as a hundred CSCs were able to form tumors in mice, whereas tens of thousands of cells 
with alternate phenotypes failed to form tumors [16]. Additional studies on brain and colon cancers also identified such subpopulations of rare cells that produce tumors in vivo $[17,18]$.

In this context, even though clinicians often observe tumor shrinkage at the first stages of multimodal treatment, often remaining resistant clones of CSCs survive and eventually cause tumor relapse and very aggressive tumor types with limited treatment alternatives [19-21]. According to the hierarchic model, only the complete abolition of CSCs can eradicate a tumor [22,23]. Thus, considerable efforts have been invested to find what distinguishes CSCs from normal stem cells and/or DCCs, to allow specific therapeutic targeting of CSCs without affecting normal cells. However, it has not been possible so far to find a marker that would solely identify cancer cells with 'stem' characteristics in distinctive tumors and tumor cell lines. Most markers identified in some cell lines that segregated cells with tumor-initiating capacity were not confirmed in other cell lines, even from the same origin. Several CSC markers have been discarded since cells expressing them have not been able to show higher tumor-initiating capacities than other tumor cells. Often, identified markers distinguish cells of mesenchymal characteristics (i.e., $\mathrm{CD} 44^{+} / \mathrm{CD} 24^{-}$in breast cancers) that confer tumor cells' more aggressive potential and the ability to induce metastatic growth. These markers, however, are not useful in tumor cell lines of mesenchymal origin. Some additional inconsistencies of the hierarchic model came from the observation that in some cases, even if CSCs were successfully eradicated, tumor growth and spread was evident [24,25]. The original CSC hypothesis has been further challenged by studies demonstrating that in some leukemias, more mature progenitor cell types or even cells expressing lineage markers can serve as leukemia-initiating cells as well [26-28].

These discrepancies raise the possibility that tumor-initiating cells might also come from differentiated cells that reacquire self-renewal capabilities [29]. Accordingly, an alternative stochastic model of tumorigenesis has been suggested. This model postulates that under a permissive environment, every cancer cell has the ability to selfrenew and can recapitulate the phenotype of the disease because of the possibility of interconversion among cell phenotypes [30-33]. Indeed, under stress conditions or after eradication of the CSC repository, some DCCs can switch their phenotype into a stem-like phenotype to ensure tumor survival and progression [15,30,34-36].

In cell cultures in attachment conditions, CSCs divide asymmetrically, reaching a final steady state with a stable CSC population rate characteristic for each cancer cell line. In addition, when DCCs are plated alone, CSCs appear after a few days in culture to regenerate the CSC population [24]. In low attachment conditions, where DCCs are not able to survive, these cells can also undergo de-differentiation to ensure survival. However, when cell culture conditions are again favorable (i.e., in attachment), the equilibrium of CSCs versus DCCs is re-established. This is accompanied by an adjustment of the stemness gene expression and invasive potential of these cells. Accordingly, de-differentiated cells in low attachment conditions rapidly express high levels of CSC genes, whereas in attachment conditions, the stemness expression signature of these DCCs is reverted. Similarly, cells cultured in low attachment are more invasive [24]. DCC to CSC reversion can be also observed in vivo [24,37]. There is evidence that intestinal epithelial cells and hepatocytes have CSC potential in colon and liver cancer models, respectively [24,38,39].

Of note, this plasticity process seems much more common than previously believed. In fact, a similar feature also occurs with healthy differentiated cells, which are known to reach a stemness state under traumatic conditions [40].

Importantly, since tumors are composed of various cell types and not only of cancer cells, the interconversion does not affect only DSCs and CSCs. In fact, a complex tumoral microecosystem that includes immune cells and stromal cells exists within a tumor, forming the so-called tumor microenvironment (TME) [41]. Cancer cells directly interact with other cells within the TME through paracrine signaling and/or direct cell-to-cell contact signaling to create a favorable physical and molecular signaling environment, to ensure tumor progression [42].

Cancer cells interact with other cells through a combination of mechanical interactions, enzymatic modification of the structure of the extracellular matrix (ECM) and signaling interactions, all of which have an effect on tumor progression and the dissemination of cancer cells (Figure 1) [43,44]. To acknowledge all of these important players, recently a new dynamical tumor initiation model for a mixed population has been proposed. It involves CSCs, DCCs and other cell types, such as quiescent cells, immune cells and fibroblasts, among others (Figure 1) [45]. In this dynamic model, each cell population becomes a nonlinear dynamical system based on its own proliferation rates controlled by chemical and/or biological pathways, and physical interactions. The dynamics are governed by the possibility of full tumor extinction, but also of overshooting dynamics after a period of remission or postsurgical relapse [45]. Importantly, implementation of the realistic complexity of tumor growth regulation changed the view of its effective eradication. Based on the dynamical tumor initiation model, the most promising strategies to fight cancer spread should be the ones that target the activator of the CSC phenotype rather than CSCs because of the feedback loops between CSCs and DDCs. New therapeutics (including nanomedicines) should thus emerge in 

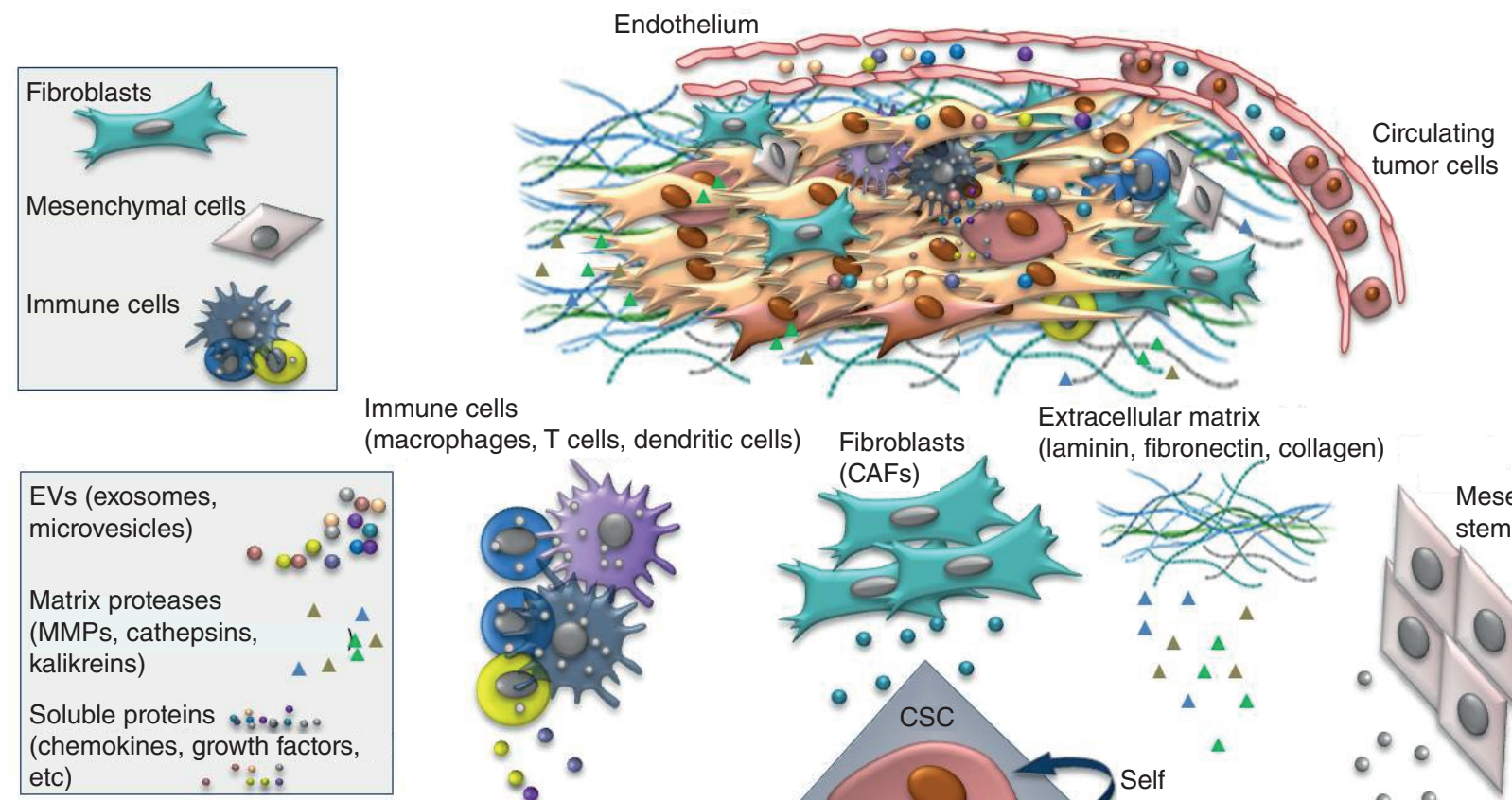

Immune cells

(macrophages, T cells, dendritic cells) Fibroblasts

Extracellular matrix
(laminin, fibronectin

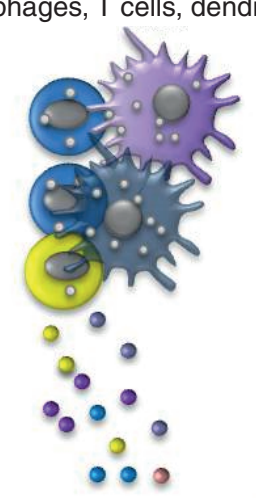
, collagen)

EVs (exosomes,
microvesicles)
Matrix proteases
(MMPs, cathepsins,
kalikreins)
Soluble proteins
(chemokines, growth factors,
etc)
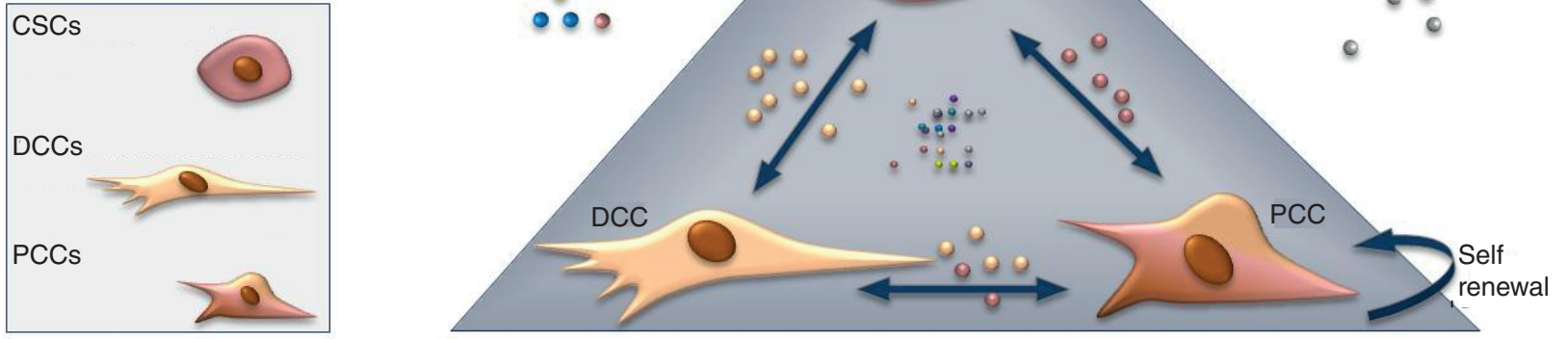

Figure 1. Dynamic cancer stem cell model. A tumor is a highly complex heterogenic dynamic entity that involves cellular components (CSCs, DCCs, fibroblasts, immune cells, mesenchymal cells, etc), cell-secreted functional molecules (growth factors, hormones, metabolites, cytokines, chemokines, etc), EVs (exosomes, vesicles, etc) and the extracellular matrix. Cancer cell subpopulations (CSCs, DCCs, PCCs) within tumors are characterized by their respective densities and rates, and the alteration of these densities and rates alters the equilibrium state of the tumor as a whole biological system. Self-renewal and differentiation rates of cancer cells are finely tuned by all tumor microenvironment players (fibroblasts, immune cells, mesenchymal cells, the extracellular matrix) to ensure tumor progression. CAF: Cancer-associated fibroblast; CSC: Cancer stem cell; DCC: Differentiated cancer cell; EV: Extracellular vesicle; PCC: Pluripotent cancer cell.

this direction $[46,47]$. With numerical simulations, the model highlights how modifications of the environment, in which the tumor expands, can abruptly change its long-time behavior [45].

\section{Regulation of phenotypic dynamism via intratumoral communication}

A role for extracellular vesicles

A tumor should be regarded as a highly complex heterogenic dynamic entity that evolves over time, best adapting to its local microenvironment and the immune system to ensure its survival. Cell-to-cell interactions, affecting CSCs' dynamic phenotype and tumor immunological response, should thus be carefully considered when proposing new anticancer nanomedicine-based therapies. Indeed, tumors from patients with recurrent resistant tumors show higher numbers of CSCs [48]. This clearly suggests the need to develop new effective treatments targeting CSCs, since CSCs account for therapy resistance and represent a cellular depot responsible of tumor regrowth and its metastatic spread [11]. Moreover, modifiable immune cells at the niches also have an important role in tumor spread, cell adaptability and survival [49]. 

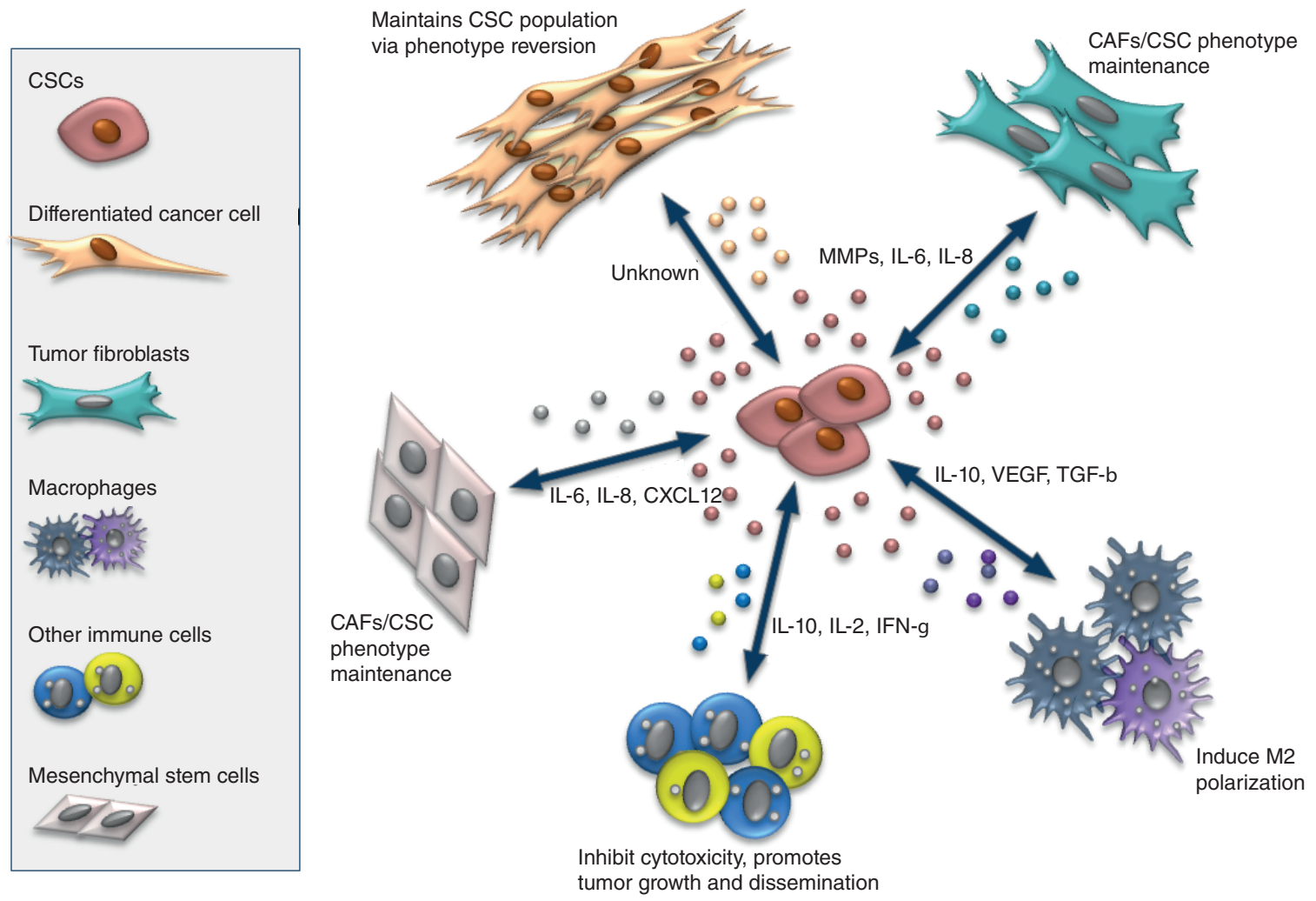

Figure 2. Signaling within the tumor niche. Complex pathways are necessary for the maintenance of the homeostasis of the CSC population. All cellular components (CSCs, DCCs, fibroblasts, immune cells, mesenchymal cells, etc.) of the tumor niche perceive the modulator effects of EVs and soluble proteins emitted by CSCs, resulting in exasperated functional capabilities. In turn, other cells from the TME produce paracrine signaling to ensure CSC growth and dissemination.

CAF: Cancer-associated fibroblast; CSC: Cancer stem cell.

Both cellular and noncellular components of the tumor niche contribute to maintain the required equilibrium and to regulate cellular plasticity [50,51]. Within the tumor niche co-exist cellular components (CSCs, DCCs, fibroblasts, immune cells, mesenchymal cells, etc), cell-secreted functional molecules (growth factors, hormones, metabolites, cytokines, chemokines, etc), extracellular vesicles (EVs; exosomes, vesicles, etc) and a sophisticated ECM that take part in integrating multiple external and internal signals to calibrate self-renewal and differentiation rates of CSCs (Figure 1) [52]. These complex pathways are essential for the maintenance of the homeostasis of the CSC population. In this regard, cell subpopulations within tumors are characterized by their respective densities and rates, and the alteration of these densities and rates alters the equilibrium state of the tumor as a whole biological system. These alterations immediately initiate the process of cell phenotypic interconversion to maintain the balance. All cellular and molecular components of the tumor niche receive the modulator effects of CSCs, resulting in exasperated functional capabilities, which in turn produce a propitious environment for CSC growth and dissemination (Figure 2).

Nonetheless, the exact molecular mechanisms that control the dynamic phenotype of CSCs still remain widely unknown. In melanoma, maintenance of the slow-cycling (JARD1B-positive) stem cell subpopulation has been proved to be dynamic. Sorted JARD1B positive cells (CSCs) gave a rise to JARD1B-negative cells, and, conversely, a single JARD1B-negative cell (DCC) originated in a heterogeneous progeny including JARD1B-positive cells [53]. Similarly, in breast cancer, it was demonstrated that basal-like mammary epithelial cells are able to spontaneously de-differentiate into stem-like cells [54]. This further reinforces the hypothesis of the existence of a controlled balance 
between CSC and DCC populations, and suggests that cancer cells opt for de-differentiation as a survival mechanism under stress conditions (i.e., chemotherapeutic treatments) [30,34]. In fact, it has also been shown that several external factors, cell interactions and paracrine cell-to-cell communication are involved in the regulation of this process. As an example, in bladder cancer, prostaglandin E2 that is secreted by apoptotic cancer cells after chemotherapy promotes proliferation of adjacent CSCs [55]. Similarly, in breast cancer, IL-6 is secreted by DCCs to induce CSCs, to maintain this dynamic equilibrium via IL-6-JAK1-STAT3 signaling [56]. Further, various aspects like cell pluripotency, invasion, proliferation, differentiation, tumor recurrence and resistance to apoptosis/treatment, among others, are regulated by constant paracrine cell-to-cell communications in both tumors and metastatic sites $[13,57]$. Late evidence suggests that this communication is at least partially mediated by EVs (Figure 2).

EVs encompass a very heterogeneous population of lipoproteic vesicles that are released into the extracellular space [58]. The main subtypes of EVs are microvesicles (MVs), exosomes and apoptotic bodies. EV subtypes differ substantially in their origin and biogenesis and thus also in their composition and size. MVs are formed by direct outward budding of plasma membrane, whereas exosomes are formed by an inward budding of the early endosomes. The maturation into multivesicular bodies is necessary during this process. However, apoptotic bodies are formed by a separation of the plasma membrane from the cytoskeleton as a result of an apoptotic process, during which dying cell contract due to increased hydrostatic pressure [59].

Although specific features have been proposed for each EV subpopulation, the identification of specific markers of each subgroup is still under debate [60]. Recently, the minimal feature requirements regarding EV characterization and a guideline to standardize studies performed with EVs have been summarized and consented [61]. Exosomes, MVs and apoptotic bodies are currently categorized based on size (small, medium or large), density (low, medium or high) and some specific markers (Alix, TSG101, HSC70 and HSP90ß) [61].

Due to their biogenesis, EVs may contain a high variety of molecular cargo of their cell of origin, such as DNA, proteins, mRNA, microRNA and lipids, which can affect multiple signaling pathways inside target cells [62]. Subsequently, EVs may trigger phenotypic changes after the interaction with their target cells, whereas single molecules that bind to a single receptor at the cell surface would often activate a single molecular pathway. Indeed, tumor cells secrete EVs bearing immunosuppressive molecules and facilitate the immunogenic escape of a growing tumor [63]. For these reasons, EVs are now considered as leading actors of the intercellular communication signaling, mediating both physiological and pathological responses.

Within EVs, big interest is centered toward exosomes, as it was shown that exosomes participate in cellto-cell communication, cell maintenance and tumor progression. In addition, exosomes have been found to stimulate immune responses by acting as antigen-presenting vesicles [58,64]. Importantly, the contents of the exosomes are not a random array of signaling molecules from undergoing intracellular events but a specific set of proteins, genetic information and lipids that essentially come from the plasma membrane and the cytosolic compartment, with very limited amounts of compounds from other intracellular compartments, such as the nucleus, the endoplasmic reticulum and the Golgi apparatus. This makes exosomes clearly different from other membrane vesicles (i.e., vesicles released by apoptotic cells). Thus, the so-called hEXO motif (an RNA-binding protein) that is responsible for miRNA sorting toward exosomes via direct biding has been recently identified [65]. Further, Wani and Kaul reported that miR-2909 is packaged in tumor exosomes by a specific 30-end post-transcriptional modification of the miRNA [66]. However, knowledge regarding exosome constitution is still missing, and strong efforts are currently devoted to unraveling how to target specific content to exosomes and EVs.

In 2007, a group led by Jan Lötvall revealed that mRNA and/or microRNA from EVs (exosomes) could be translated into proteins in target cells, providing the first demonstration of a genetic information cell-to-cell transfer mediated by exosomes [67]. Equally, EVs released by embryonic stem cells can reprogram hematopoietic progenitors by horizontal transfer of specific mRNA and proteins. Comparable 'information exchange' represents one of the critical components of conditioning premetastatic niches and is involved in the control of CSC selfrenewal and tumor expansion. Released EVs can directly stimulate surrounding cells by interacting with specific surface receptors or transferring from one cell to another various bioactive molecules, including membrane receptors, proteins, mRNA, microRNA and even whole organelles (Figure 1).

The colonization by metastatic cells requires the existence of a hospitable microenvironment in distal sites that allows an adequate hosting of the metastatic arriving cells, fostering their survival. Accordingly. EVs derived from tumor cells can remain at the extracellular space, can remain at the site of origin or can enter into biological fluids, reaching distal sites and participating in the creation of premetastatic niches. Moreover, exosomes from other cells involved in the TME (i.e., fibroblasts, stromal cells, immune cells) influenced CSC-DCC balance in a bidirectional 
paracrine setting (Figure 2). Fibroblasts have been reported to secrete diverse bioactive factors such as cytokines (IL-6, IL-8) that can mediate the conversion of DCCs into CSCs [68]. Further, the communication between tumor cells and stromal cells in the metastatic niche, mediated by EVs released by the primary tumor, has a critical role in facilitating cancer metastasis. It has been shown based on specific expression patterns of integrins and glycans that tumor-derived EVs are uptaken by tissue-specific cells to prepare premetastatic niches $[69,70]$.

Furthermore, the resistance of tumor cells to chemotherapeutic treatments also involves cellular intercommunication mediated by EVs, as intratumoral CSC/DCC content is relevant to define the treatment response and recurrence of tumor. Released $\mathrm{EV}$ s evacuate pro-apoptotic proteins or directly withdrawn therapeutic drugs from cells. Indeed, resistant CSCs release significantly more EVs than cells responsive to antitumor therapy. Moreover, EVs also appear to favor CSC survival by reducing the responsiveness of T cells or directly inducing apoptosis in activated antitumor $T$ cells [57]. In fact, fibroblasts and immune cells at the tumor niches are substantially involved in tumor spread, adaptability and survival [49]. Understanding the tight relationship among CSCs, the TME and the immune response is essential for developing effective anticancer nanomedicine-based treatments (Figure 2).

\section{EVs as natural nanocarriers engaged in cell-to-cell intercommunication}

Despite that nanomedicine is regarded as one of the most promising fields of research to bring new treatment strategies in cancer care, only very few $(2 \%)$ nanocarrier-based treatments have successfully entered into clinical trials. Moreover, only a limited handful (15) of these formulations have reached clinical practice [4]. There are a variety of reasons for this still suboptimal translation from bench to bedside, like inadequate physicochemical features of nanomaterials, improper preclinical efficacy and safety, and regulatory and manufactory issues. Further, changes of the properties and complexity of the nanomaterials when in biological fluids (i.e., protein corona, cargo release, opsonization) are difficult to compute and significantly alter the functionality of nanomedicines after administration [71]. There are multiple factors that modulate the toxicity of nanomaterials, such as size, shape, porosity and surface charge. Another major hurdle is the feasibility of manufacturing nanomedicines under good manufacturing practices. Although most clinical and preclinical studies have been made from small amounts of materials, large-scale production requires great effort to maintain their physicochemical properties and release capabilities. Currently, a lot of effort is put into amelioration of large-scale synthesis (i.e., CELLine Bioreactor Flask) that is required for the EV's clinical translation. The other option is the production of synthetic EV-based drug delivery systems (DDS) that could be produced as a large-scale homogeneous population with standardized protocols [72]. In this regard, EVs demonstrate clear prospects as nanocarriers, as they have a biological origin, little toxicity and lack of immunogenicity [73]. EVs are also very promising as delivery systems due to their capacity to directly release their cargo into the cellular cytoplasm of targeted cells. After internalization, EVs can also fuse with the endosomal membrane and release their content $[58,64]$. Their structure resembles that of liposomes, but their lipid layer has a more complex composition. They are characterized by the presence of multiple families of proteins such as tetraspanins (CD63, CD81, CD9), heat shock proteins (HSC70), lysosomal proteins (Lamp2p) and fusion proteins such as CD9, flotillin and Annexin (Figure 3). The complexity of their membrane composition may help EVs escape endosomes, thus allowing delivery of material into the nucleus, whereas artificial nanocarriers will require complex protein and peptide modifications [74]. However, EVs are known to be nonimmunogenic, which is certainly an advantage to reduce potential treatment resistance if using protein-based therapies, particularly as enzyme replacement therapies. Moreover, EVs might be the best option when dealing with nondruggable target candidates. Further, one of the challenges of drug delivery systems is to optimize their targeting properties to release the therapeutic compounds only in specific organs/tissues of the body, avoiding or reducing systemic toxicity. In this regard, there is strong data in the literature reporting preferential delivery of EVs (exosomes) to specific target tissues according to their integrin and glycan receptor profile of their membrane surface [69,70]. This is one of the reasons that might explain why EVs from different tumor types have a tendency to modify and condition specific distal tissues to host tumor cells as metastatic niches for each particular tumor type.

In this context, modifying EV-producing cells may lead to the design of EVs with adequate profiles that are required to target specific tissues. Previously, EVs donor cells were transfected with pDisplay ${ }^{T M}$ encoding the transmembrane domain of platelet-derived growth factor receptor fused to a peptide that specifically binds to the EGFR. Subsequently, released EVs efficiently delivered miRNA to EGFR+ cancer cells [75]. Alternatively, to further enhance the display of targeting peptides on the EV's surface, a donor cell may be transected with the engineered vectors that contain a gene of a protein of the exosomal membrane (e.g., Lamp2b) fused with targeting peptides. EVs released from such modified cell lines will display the targeting peptides on their surface [76-78]. 


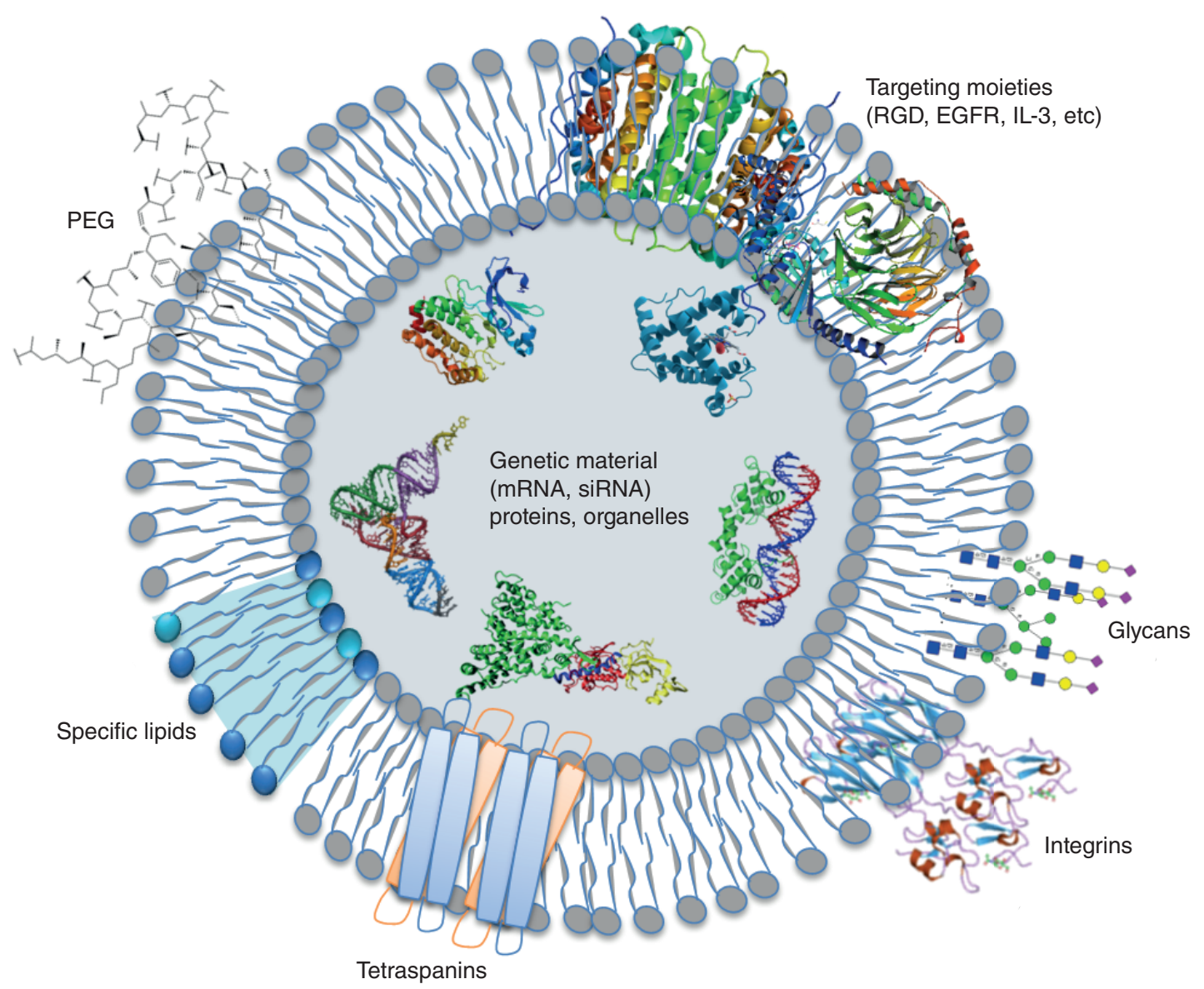

Figure 3. Structure of extracellular vesicles. Extracellular vesicles are lipid vesicles with a complex membrane composition. They express multiple families of proteins, such as tetraspanins (CD63, CD81, CD9), heat shock proteins (HSC70), lysosomal proteins (Lamp2p) and fusion proteins, such as CD9, flotillin and annexin, at the membrane. Within the inner core, they may contain mRNA, miRNA, proteins and organelles, among others.

This approach was first used by Alvarez-Erviti et al. to produce EVs displaying the neuron-specific RVG peptide on their surface [79]. Importantly, the authors demonstrated brain-targeting capability of the engineered EVs that delivered stable siRNA after systemic administration in mice. A similar approach has been used to deliver imatinib and siRNA to leukemic cells, using EVs with overexpressed IL-3 on their surface [80].

Another way to tailor-make EV surface targeting peptides is through membrane functionalization by covalent modification. Accordingly, this technique modifies directly isolated EVs rather than modifying their cells of origin. In this regard, EVs with both imaging and therapeutic properties by loading paramagnetic iron oxide nanoparticles and curcumin have been proposed for a glioblastoma theranostic approach. Targeting of glioma has been increased by adding the neuropilin-1-targeted peptide to the exosomal membrane by click chemistry [81]. Alternatively, the membrane of $\mathrm{EVs}$ was functionalized by a cationic lipid and $\mathrm{pH}$-sensitive peptide (GALA) conjugation to improve EV cellular uptake and cytosolic release [82]. It was also demonstrated that it is possible to increase the specificity of EVs to target cancer cells through an RNA nanotechnology approach. To this aim, cholesterolconjugated RNA aptamers harboring a specific targeting domain for cancer cells were developed. Resultant EVs conjugated with RNA nanoparticles harboring EGFR aptamers and loaded with survivin siRNA were able to inhibit breast cancer growth in mice [83]. Some examples of successful preclinical EV development are summarized in Table 1. Nonetheless, even though EVs have demonstrated clear prospects as natural delivery systems, many 
Table 1. List of modified extracellular vesicles designed to alter the tumor microenvironment.

\begin{tabular}{|c|c|c|c|c|}
\hline Extracellular vesicle source & Extrinsic cargo & Extrinsic targeting & Cancer type & Ref. \\
\hline Bone marrow-derived MSCs & TRAIL & $\mathrm{N} / \mathrm{A}$ & Various cancers & [84] \\
\hline Various carcinoma cells & Doxorubicin, cisplatin, methotrexate & $\mathrm{N} / \mathrm{A}$ & Various cancers & [85] \\
\hline B16-F10 melanoma cells & SPIONs & $\mathrm{N} / \mathrm{A}$ & Melanoma & [86] \\
\hline LNCaP, PC-3 cancer cells & Paclitaxel & $\mathrm{N} / \mathrm{A}$ & Prostate cancer & [87] \\
\hline Immature dendritic cells & Doxorubicin & iRGD-Lamp2b & Breast cancer & [88] \\
\hline Bovine milk & Paclitaxel & $\mathrm{N} / \mathrm{A}$ & Lung cancer & [89] \\
\hline H22 hepatocarcinoma cells & Doxorubicin, 5-FU & $\mathrm{N} / \mathrm{A}$ & Hepatocarcinoma & [91] \\
\hline Raw 264.7 macrophages & Doxorubicin & DEAP & Colon cancer & [92] \\
\hline HeLa cervical cancer cells & Dextran & GALA & Cervical cancer & [82] \\
\hline Primary dendritic cells & VEGF siRNA & $\begin{array}{l}\text { AS1411 aptamer (nucleolin } \\
\text { targeting) }\end{array}$ & Breast cancer & [93] \\
\hline Mesenchymal stromal cells & Paclitaxel & $\mathrm{N} / \mathrm{A}$ & Pancreatic cancer & [95] \\
\hline Red blood cells & miR-125b-2, Cas mRNA & $\mathrm{N} / \mathrm{A}$ & Leukemia, breast cancer & [96] \\
\hline Plants (grapefruit) & miR-17, miR18s & $\mathrm{N} / \mathrm{A}$ & Brain and colon cancer & {$[97,98]$} \\
\hline
\end{tabular}

aspects of their stability, integrity and reproducibility need to be considered during scale-up production. In fact, the great interest and expectations toward the use of EVs as nanocarriers is hampered by a current low-production rate to high-production cost ratio. In addition, some regulatory aspects regarding the clinical use of modified EVs have not been entirely defined. Specific guidelines and regulation regarding EV isolation and characterization (content, surface, isolation method) is thus required to speed up the translation from bench to bedside of EV-based nanomedicines.

\section{Nanomedicines to modulate the tumor microenvironment \& reduce tumor spread}

As mentioned earlier, the interaction between stromal and tumor cells within a specific TME plays a critical role in tumor growth and dissemination. Cancer cells require mechanical support, blood supply and growth factors secreted by stromal cells to guarantee continuous growth [99]. In fact, the initiation of tumor growth and metastatic growth is linked with the alteration of the healthy stroma and the appearance of a pro-tumoral environment (i.e., inflammatory signatures). This process includes neoangiogenesis, infiltration of fibroblasts and immune cells, and extensive remodeling of the ECM, and exhibits strong similarities to normal wound healing [100]. Cancer stroma thus not only supports but rather directs cancer progression and dissemination.

The most abundant cells of the tumor stroma are cancer-associated fibroblasts (CAFs), which significantly influence cancer growth and progression through TME control [101]. As mentioned previously, a bidirectional paracrine communication between CAFs and CSCs/DCCs is indispensable to maintain the required balance of cell populations within the TME. Of note, normal fibroblasts are activated into overproliferative CAFs by sonic hedgehog proteins that CSCs secrete into the niche. These in turn activate CAFs via upregulation of the WNT/hedgehog signaling pathway [102]. However, CAFs are the main source of multifunctional cytokines (IL-6, IL-8) that regulate CSC-DCC balance and neoangiogenesis within the TME [103-105]. Importantly, paracrine $\mathrm{CAF} /$ tumor cell signaling could also be mediated by EV signal transfer as described previously. In addition, CAFs secrete a variety of growth factors (i.e., transforming growth factor $\beta$ (TGF $\beta$ ), vascular endothelial growth factor and platelet-derived growth factor, interleukins and ECM components (i.e., collagens, fibronectins and metalloproteinases) that support tumor growth, generate a physical barrier against drugs and immune infiltration, and facilitate cancer invasion [101,103-105]. Indeed, CAFs may directly influence tumor infiltrating immune cells and modulate the immune microenvironment. 
Table 2. List of alternative nanomedicines that modulate the tumor microenvironment/immune system.

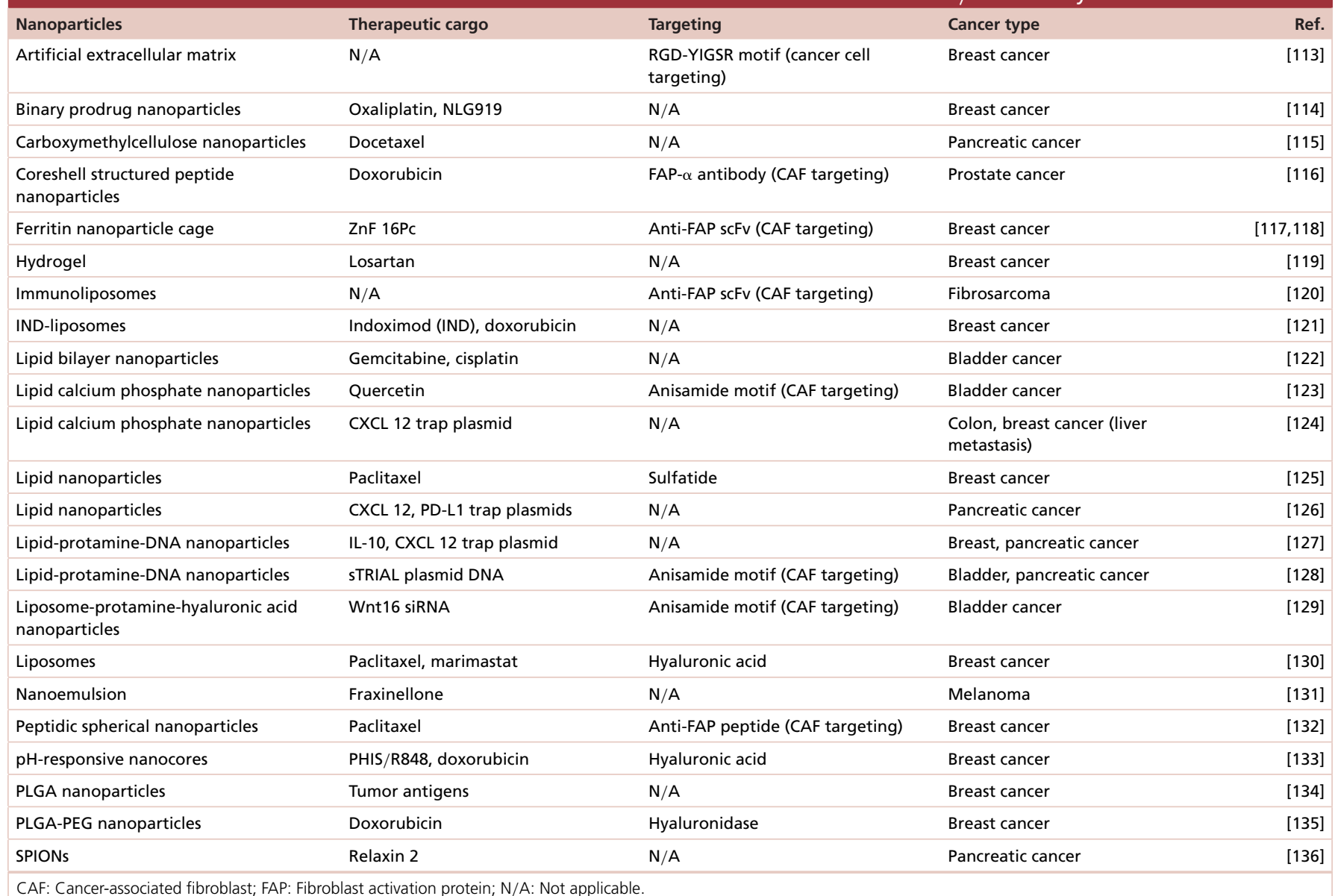

Activated CAFs express TGF- $\beta, \alpha$-SMA, FAP, PDGFR- $\alpha / \beta$ and S100A4, among others. After activation, CAFs fail to reacquire an inactive status, and therefore they constantly exert a pathophysiological response producing a desmoplastic TME. Further, CAFs may induce epithelial to mesenchymal transition by secreting carbonic anhydrase IX, which causes acidification of the ECM and further enhances the activity of MMP-2 and MMP-9 metalloproteases, which in turn facilitates the local invasion of cancer cells into their surrounding tissues [106]. This has been observed in pancreatic cancer, prostate cancer, squamous cell cancer and melanoma, although with a variable degree of desmoplasia [107-110]. Because of this, new strategies that either reduce stromal desmoplasia or inactivate CAFs have been explored over the past years (Table 2). In this regard, a recent study showed that the anticancer compound minnelide is able to disrupt TGF- $\beta$ signaling and revert activated CAFs into a quiescent form by decreasing $\alpha$-SMA expression. Moreover, the application of all-transretinoic acid promotes a quiescent form of pancreatic stellate cells by modulating Wnt- $\beta$ cathenin signaling, reducing tumor progression [111]. Furthermore, hydrophobic drugs with activity against CAFs have been also encapsulated into nanocarriers to improve their solubility. Accordingly, various types of nanocarriers with distinct functions have been proposed against CAFs, including liposomes, polymeric nanoparticles, nanoemulsions and inorganic nanoparticles. In addition, surface moieties have been conjugated to nanocarriers that have been modified to increase their stability in circulation and to enhance targeting efficiency (Table 2). Alternative strategies have been also tested to modify the ECM to enhance the tumor penetration of drugs while reducing metastatic spread of the disease. An example is the use of a PEGylated recombinant formulation of human hyaluronidase (PEGPH20) aimed to improve the tumor penetration efficiency of chemotherapeutics. In fact, hyaluronic acid is a major component of the ECM and increases the interstitial pressure inside tumors. Hyaluronic acid degradation by PEGPH20 normalizes the interstitial pressure within the tumor and enhances the delivery of cytotoxic agents. In this context, PEGPH20 has been successfully tested in combination with cytotoxic drugs in clinics (i.e., nab-paclitaxel/gemcitabine and doxorubicin). Although phase 
I-II studies showed promising results in patients with high hyaluronic acid-expressing tumors, the phase III study of PEHPH20 was not as successful and thus its further development is currently on pause. Additional intrinsic factors, such as lack of significant neoantigens, low tumor mutational burden, and epithelial to mesenchymal transition, should be considered [112]. Currently, PEHPH20 is still tested in combination with pembrolizumab (MK-3475) for patients with previously treated hyaluronan-high metastatic pancreatic ductal adenocarcinoma (NCT03634332) and in combination with FOLFIRINOX in patients with newly diagnosed metastatic pancreatic cancer (NCT01959139).

Recently, in addition to chemotherapy, nanocarriers have also been designed to improve the efficiency of cancer immunotherapy. Lipid-calcium-phosphate (LCP) nanoparticles have been successfully used as a peptide vaccine delivery system to stimulate anticancer $\mathrm{T}$-cell response [137]. Another strategy has elegantly combined an LCPbased vaccine with targeted silencing of TGF- $\beta$ expression at the TME using liposome-protamine-hyaluronic acid nanoparticles. Since TGF- $\beta$ acts as immune suppressor within the TME, its silencing enhances the systemic immune response against the tumor. A combination of these two nanoparticles offers a flexible and powerful promising platform for immunotherapy [138]. In this regard, nanoparticles have been also conjugated to the surface of transferred T cells, acting as persistent autocrine-like signals to stimulate the immune response against tumors [139]. Further, inflammation regulators such as cytokines have been loaded into CAFs by using targeted nanoparticles to modify the TME $[131,140,141]$. Another example is the use of a PEGylated liposome (LPD) with a plasmid-encoding CXCL12 fusion protein (CXCL12 trap), combined with PD-L1. CXCL12 trap facilitates the penetration of T cells into the TME, whereas PD-L1 trap allows infiltrated T cells to kill the cancer cells. A similar setting of lipid-coated calcium phosphate nanoparticles (LCP) also resulted in enhanced infiltration of T lymphocytes while causing a drop in the expression of CXCL12 and immunosuppressive cells in the liver, clearly suggesting a potential impact of nanomedicine in modulating anticancer immune response [126].

Overall, the list of alternative nanomedicines that have been successfully explored in preclinical settings as $\mathrm{TME} /$ immune system modulators to reduce tumor progression and metastasis is increasing (Table 2). There is no doubt that in the future, new nanomedicine-based strategies against cancer should focus on the use of nanomedicine to therapeutically intervene in the different aspects that define a tumor and its microenvironment, as well as in the immunological response of the immune system to combat metastasis and the preconditioning of metastatic sites.

\section{Conclusion}

A tumor should be regarded as a heterogeneous ensemble of different cell types that are in constant intercommunication to orchestrate the growth and dissemination of the tumor. In addition, cells with metastatic potential communicate with distant tissues to prepare niches for hosting and nesting cancer cells to allow spread of the disease. According to the current dynamic CSC model of tumor growth, new nanomedicine-based therapies under development should focus not only on eradicating cancer cells but should also consider all components of the TME. In fact, after the eradication of the CSC population, specific complex mechanisms (feedback loops, phenotypic reversion and paracrine messaging) become active to re-establish the cellular balance within the TME and guarantee the progression and dissemination of the tumor. In this scenario, a clear understanding of the information exchanged between CSCs and differentiated cells (DCCs) to surrounding cells and, further, the understanding of close interactions among players within the metastatic niche are of utmost importance to develop efficient treatments.

\section{Future perspective}

According to the state of the art exposed in this review, a deep characterization of the content from secreted EVs as the main responsible actors of the cell-to-cell intercommunication within the tumors and metastatic sites is needed. Deciphering of EV content would lead to a better understanding of the fundamental nature of CSC/DCC crosstalk, the reversion phenotype of DCCs into CSCs, and their impact on immune response, tumor recurrence, metastasis and future clinical treatment outcomes. Similarly, to decoding ENIGMA during World War II that helped allied troops decipher encoded messages from enemy forces and prompted the end of the conflict, EV cargo will provide new candidate targets for future clinical interventions. Deciphering messages emitted by cancer cells and using nanomedicine-based strategies to modulate these messages should counteract metastatic dissemination and treatment resistance, which are the principal causes of mortality of oncologic patients worldwide. Likewise, a better understanding of the immunological processes occurring at the TME should also open the possibility to design new strategies to combat cancer relapse and metastatic spread. 
Moreover, standardized protocols for isolation, modification and loading of EVs are required to prompt EV use in clinical practice. Current lack of quality controls under good manufacturing practices for the evaluation of safety and functionalization of large-scale EV production is preserving their use as DDS.

For all of these reasons, it is necessary to gain insight into fundamental processes of EV biology, to understand the basic mechanisms through which these vehicles can target specific cell types and their precise roles in the TME and develop new methodologies to translate their potential as DDS into clinics. This new knowledge will definitely help in further improving EVs for drug delivery in cancer.

Executive summary

Cancer stem cell models (the hierarchic model, stochastic model \& dynamical tumor initiation model)

- A tumor should be regarded as a highly complex heterogenic dynamic entity that evolves over time, best adapting to its local microenvironment and the immune system to ensure its survival.

- The dynamic tumor initiation model for a mixed population describes the cancer stem cell (CSC) dynamic phenotype and cancer initiation.

Regulation of phenotypic dynamism via intratumoral communication

- Cancer cells directly interact with other cells within the tumor microenvironment (TME) through paracrine signaling and/or direct cell-to-cell contact signaling to create a favorable physical and molecular signaling environment, to ensure tumor progression.

- Extracellular vesicles (EVs) play an important regulatory role in TME modulation and CSC dynamics.

EVs as natural nanocarriers engaged into cell-to-cell intercommunication

- EVs and nanomedicines designed to modulate the TME should be regarded as effective anticancer therapy rather than CSC elimination.

Nanomedicines to modulate the TME \& reduce tumor spread

- To inhibit tumor growth and dissemination, nanomedicines should directly target cancer cells and should also modulate the TME.

Author contributions

P Gener drafted the work and figures. P Gonzalez Callejo, J Seras-Franzoso, F Andrade and D Rafael contributed to the writing, and I Abasolo and S Schwartz Jr revised it critically for important intellectual content and approved the final version.

Financial \& competing interests disclosure

This work was partially supported by grant PI17/02242 from Fondo de Investigaciones Sanitarias (FIS) of Instituto Carlos III (ISCIII), co-financed by the European Regional Development Fund (FEDER), and EvoNano project, funded by European Union's Horizon 2020 FET Open Programme under grant agreement no. 800983. The authors have no other relevant affiliations or financial involvement with any organization or entity with a financial interest in or financial conflict with the subject matter or materials discussed in the manuscript apart from those disclosed.

No writing assistance was utilized in the production of this manuscript.

\section{Open access}

This work is licensed under the Attribution-NonCommercial-NoDerivatives 4.0 Unported License. To view a copy of this license, visit http://creativecommons.org/licenses/by-nc-nd/4.0/

\section{References}

Papers of special note have been highlighted as: $\bullet$ of interest

1. Capp JP. Cancer stem cells: from historical roots to a new perspective. J. Oncol. 2019, 5189232 (2019).

2. Dixon FJ, Moore RA. Testicular tumors; a clinicopathological study. Cancer 6(3), 427-454 (1953).

3. Bonnet D, Dick JE. Human acute myeloid leukemia is organized as a hierarchy that originates from a primitive hematopoietic cell. Nat. Med. 3, 730-737 (1997).

- $\quad$ First article describing the existence of cancer stem cells.

4. Lapidot T, Sirard CF, Vormoor JF et al. A cell initiating human acute myeloid leukaemia after transplantation into SCID mice. Nature 367(6464), 645-648 (1994).

5. Dionne L, Driver E, Wang X. Head and neck cancer stem cells: from identification to tumor immune network. J. Dent. Res. 94(11), 1524-1531 (2015). 
6. Hermann PC, Bhaskar S, Cioffi M, Cioffi MF, Heeschen C. Cancer stem cells in solid tumors. Semin. Cancer Biol. 20(2), 77-84 (2010).

7. Huang R, Rofstad EK. Cancer stem cells (CSCs), cervical CSCs and targeted therapies. Oncotarget 8(21), 35351-35367 (2016).

8. Testa UA, Petrucci E, Pasquini LA, Castelli GF, Pelosi E. Ovarian cancers: genetic abnormalities, tumor heterogeneity and progression, clonal evolution and cancer stem cells. Medicine (Basel) 5(1), 16 (2018).

9. Testa UA, Pelosi E, Castelli G. Colorectal cancer: genetic abnormalities, tumor progression, tumor heterogeneity, clonal evolution and tumor-initiating cells. Med. Sci. (Basel) 6(2), 31 (2018).

10. Dean M, Fojo T, Bates S. Tumour stem cells and drug resistance. Nat. Rev. Cancer 5(4), 275-284 (2005).

11. Gener P, Rafael DF, Fernandez Y et al. Cancer stem cells and personalized cancer nanomedicine. Nanomedicine (Lond.) 11(3), 307-320 (2016).

12. Najafi M, Mortezaee K, Majidpoor J. Cancer stem cell (CSC) resistance drivers. Life Sci. 234, 116781 (2019).

13. Sun ZA, Wang L, Dong L, Wang X. Emerging role of exosome signalling in maintaining cancer stem cell dynamic equilibrium. J. Cell. Mol. Med. 22(8), 3719-3728 (2018).

- Describes the role of exosomes in maintaining cancer stem cell dynamic equilibrium.

14. Reya T, Morrison SJ, Clarke MF, Weissman IL. Stem cells, cancer, and cancer stem cells. Nature 414(6859), 105-111 (2001).

15. Wicha MS, Liu S, Dontu G. Cancer stem cells: an old idea—a paradigm shift. Cancer Res. 66(4), 1883-1890 (2006).

16. Al-Hajj M, Wicha MS, Benito-Hernandez A, Morrison SJ, Clarke MF. Prospective identification of tumorigenic breast cancer cells. Proc. Natl. Acad. Sci. U. S. A. 100(7), 3983-3988 (2003).

17. Ricci-Vitiani L, Lombardi DG, Pilozzi E et al. Identification and expansion of human colon-cancer-initiating cells. Nature 445(7123), 111-115 (2007).

18. Singh SK, Hawkins CF, Clarke ID et al. Identification of human brain tumour initiating cells. Nature 432(7015), 396-401 (2004).

19. Alamgeer MF, Ganju VF, Kumar BF et al. Changes in aldehyde dehydrogenase-1 expression during neoadjuvant chemotherapy predict outcome in locally advanced breast cancer. Breast Cancer Res. 16(2), R44 (2014).

20. Dzobo K, Senthebane DA, Rowe A et al. Cancer stem cell hypothesis for therapeutic innovation in clinical oncology? Taking the root out, not chopping the leaf. OMICS 20(12), 681-691 (2016).

21. Lagadec C, Vlashi E, Della DL et al. Survival and self-renewing capacity of breast cancer initiating cells during fractionated radiation treatment. Breast Cancer Res. 12(1), R13 (2010).

22. Vinogradov S, Wei X. Cancer stem cells and drug resistance: the potential of nanomedicine. Nanomedicine (Lond.) 7(4), 597-615 (2012).

23. Zhao Y, Alakhova DY, Kabanov AV. Can nanomedicines kill cancer stem cells? Adv. Drug Deliv. Rev. 65(0), 1763-1783 (2013).

24. Gener P, Rafael D, Seras-Franzoso J et al. Pivotal role of AKT2 during dynamic phenotypic change of breast cancer stem cells. Cancers (Basel) 11(8), 1058 (2019).

- $\quad$ Describes regulation of cancer stem cell reversion in vitro and in vivo.

25. Gener $\mathrm{P}$, Montero S, Xandri-Monje $\mathrm{H}$ et al. Zileuton ${ }^{\mathrm{TM}}$ loaded in polymer micelles effectively reduce breast cancer circulating tumor cells and intratumoral cancer stem cells. Nanomedicine 24, 102106 (2019).

26. Jamieson $\mathrm{CH}$, Ailles LE, Dylla SJ et al. Granulocyte-macrophage progenitors as candidate leukemic stem cells in blast-crisis CML. $N$. Engl. J. Med. 351(7), 657-667 (2004).

27. Goardon N, Marchi E, Atzberger AF et al. Coexistence of LMPP-like and GMP-like leukemia stem cells in acute myeloid leukemia. Cancer Cell 19(1), 138-152 (2011).

28. Sarry JE, Murphy KF, Perry RF et al. Human acute myelogenous leukemia stem cells are rare and heterogeneous when assayed in NOD/SCID/IL2R $\gamma \mathrm{c}$-deficient mice. J. Clin. Invest. 121(1), 384-395 (2011).

29. Reya T, Morrison SJ, Clarke MF, Weissman IL. Stem cells, cancer, and cancer stem cells. Nature 414(6859), 105-111 (2001).

30. Gupta PB, Fillmore CM, Jiang G et al. Stochastic state transitions give rise to phenotypic equilibrium in populations of cancer cells. Cell 146, 633-644 (2011).

- Describes the mathematical model of dynamic cancer equilibrium.

31. Shackleton M. Normal stem cells and cancer stem cells: similar and different. Semin. Cancer Biol. 20(2), 85-92 (2010).

32. Sell S. Stem cell origin of cancer and differentiation therapy. Crit. Rev. Oncol. Hematol. 51(1), 1-28 (2004).

33. Huntly BJ, Gilliland DG. Leukaemia stem cells and the evolution of cancer-stem-cell research. Nat. Rev. Cancer 5(4), 311-321 (2005).

34. Gener P, Seras-Franzoso J, Callejo PG et al. Dynamism, sensitivity, and consequences of mesenchymal and stem-like phenotype of cancer cells. Stem Cells Int. 2018, 4516454 (2018).

35. Kreso A, Dick JE. Evolution of the cancer stem cell model. Cell Stem Cell 14, 275-291 (2014).

36. Shackleton M, Quintana E, Fearon ER, Morrison SJ. Heterogeneity in cancer: cancer stem cells versus clonal evolution. Cell 138, 822-829 (2009). 
37. Shimokawa M, Ohta Y, Nishikori S et al. Visualization and targeting of LGR5+ human colon cancer stem cells. Nature 545(7653), 187-192 (2017).

38. Perekatt AO, Shah PP, Cheung S et al. SMAD4 suppresses WNT-driven dedifferentiation and oncogenesis in the differentiated gut epithelium. Cancer Res. 78(17), 4878-4890 (2018).

39. Tummala KS, Brandt M, Teijeiro A, et al. Hepatocellular carcinomas originate predominantly from hepatocytes and benign lesions from hepatic progenitor cells. Cell Rep. 19(3), 584-600 (2017).

40. Aponte PM, Caicedo AA. Stemness in cancer: stem cells, cancer stem cells, and their microenvironment. Stem Cells Int. 2017, 5619472 (2017).

41. McAllister SS, Weinberg RA. The tumour-induced systemic environment as a critical regulator of cancer progression and metastasis. Nat. Cell Biol. 16(8), 717-727 (2014).

42. Pietras K, Ostman A. Hallmarks of cancer: interactions with the tumor stroma. Exp. Cell Res. 316(8), 1324-1331 (2010).

43. Malik R, Lelkes PI, Cukierman E. Biomechanical and biochemical remodeling of stromal extracellular matrix in cancer. Trends Biotechnol. 33(4), 230-236 (2015).

44. Bloom AB, Zaman MH. Influence of the microenvironment on cell fate determination and migration. Physiol. Genomics 46(9), 309-314 (2014).

45. Olmeda F, Ben AM. Clonal pattern dynamics in tumor: the concept of cancer stem cells. Sci. Rep. 9(1), 15607 (2019).

\section{- Describes the dynamic cancer stem cell model.}

46. Liu T, Wu X, Chen T, Luo Z, Hu X. Downregulation of DNMT3A by miR-708-5p inhibits lung cancer stem cell-like phenotypes through repressing Wnt/beta-catenin signaling. Clin. Cancer Res. 24(7), 1748-1760 (2018).

47. Jimeno A, Gordon M, Chugh R et al. A first-in-human phase I study of the anticancer stem cell agent ipafricept (OMP-54F28), a decoy receptor for Wnt ligands, in patients with advanced solid tumors. Clin. Cancer Res. 23(24), 7490-7497 (2017).

48. Tanei T, Morimoto KF, Shimazu KF et al. Association of breast cancer stem cells identified by aldehyde dehydrogenase 1 expression with resistance to sequential paclitaxel and epirubicin-based chemotherapy for breast cancers. Clin Cancer Res. 15(12), 4234-4241 (2009).

49. Blomberg OS, Spagnuolo L, de Visser KE. Immune regulation of metastasis: mechanistic insights and therapeutic opportunities. Dis. Model Mech. 11(10), dmm036236 (2018).

50. Lee G, Hall RR, Ahmed AU. Cancer stem cells: cellular plasticity, niche, and its clinical relevance. J. Stem Cell Res. Ther. 6(10), 363 (2016).

51. Varas-Godoy MA, Rice GA, Illanes SE. The crosstalk between ovarian cancer stem cell niche and the tumor microenvironment. Stem Cells Int. 2017, 5263974 (2017).

52. Plaks V, Kong N, Werb Z. The cancer stem cell niche: how essential is the niche in regulating stemness of tumor cells. Cell Stem Cell 16(3), 225-238 (2015).

53. Roesch A, Fukunaga-Kalabis M, Schmidt EC et al. A temporarily distinct subpopulation of slow-cycling melanoma cells is required for continuous tumor growth. Cell 141(4), 583-594 (2010).

54. Chaffer CL, Brueckmann I, Scheel C et al. Normal and neoplastic nonstem cells can spontaneously convert to a stem-like state. Proc. Natl. Acad. Sci. U. S. A. 108(19), 7950-7955 (2011).

- One of the first articles describing cancer stem cell reversion.

55. Kurtova AV, Xiao J, Mo Q et al. Blocking PGE2-induced tumour repopulation abrogates bladder cancer chemoresistance. Nature 517, 209-213 (2015).

56. Kim SY, Kang JW, Song X et al. Role of the IL-6-JAK1-STAT3-Oct-4 pathway in the conversion of non-stem cancer cells into cancer stem-like cells. Cell. Signal. 25(4), 961-969 (2013).

57. Maacha S, Bhat AA, Jimenez L et al. Extracellular vesicles-mediated intercellular communication: roles in the tumor microenvironment and anti-cancer drug resistance. Mol. Cancer 18(1), 55 (2019).

58. Raposo G, Stoorvogel W. Extracellular vesicles: exosomes, microvesicles, and friends. J. Cell Biol. 200(4), 373-383 (2013).

59. Doyle LM, Wang MZ. Overview of extracellular vesicles, their origin, composition, purpose, and methods for exosome isolation and analysis. Cells 8(7), 727 (2019).

60. Meldolesi J. Exosomes and ectosomes in intercellular communication. Curr. Biol. 28(8), R435-R444 (2018).

61. Thery CA, Witwer KW, Aikawa E et al. Minimal information for studies of extracellular vesicles 2018 (MISEV2018): a position statement of the International Society for Extracellular Vesicles and update of the MISEV2014 guidelines. J. Extracell. Vesicles 7(1), 1535750 (2018).

62. Zhang HG, Grizzle WE. Exosomes: a novel pathway of local and distant intercellular communication that facilitates the growth and metastasis of neoplastic lesions. Am. J. Pathol. 184(1), 28-41 (2014).

63. Parolini I, Federici CF, Raggi CF et al. Microenvironmental pH is a key factor for exosome traffic in tumor cells. J. Biol. Chem. 284(49), 34211-34222 (2009). 
64. Isola AL, Chen S. Exosomes: the messengers of health and disease. Curr. Neuropharmacol. 15(1), 157-165 (2017).

65. Santangelo L, Giurato G, Cicchini C et al. The RNA-binding protein SYNCRIP is a component of the hepatocyte exosomal machinery controlling microRNA sorting. Cell Rep. 17(3), 799-808 (2016).

66. Wani S, Kaul DA. Cancer cells govern miR-2909 exosomal recruitment through its 3 '-end post-transcriptional modification. Cell Biochem. Funct. 36(2), 106-111 (2018).

67. Valadi H, Ekstrom KF, Bossios AF et al. Exosome-mediated transfer of mRNAs and microRNAs is a novel mechanism of genetic exchange between cells. Nat. Cell. Biol. 9(6), 654-659 (2007).

68. Wang M, Zhao J, Zhang L et al. Role of tumor microenvironment in tumorigenesis. J. Cancer 8(5), 761-773 (2017).

69. Hoshino A, Costa-Silva B, Shen TL et al. Tumour exosome integrins determine organotropic metastasis. Nature 527, 329-335 (2015).

70. Shimoda A, Tahara Y, Sawada SI, Sasaki Y, Akiyoshi K. Glycan profiling analysis using evanescent-field fluorescence-assisted lectin array: importance of sugar recognition for cellular uptake of exosomes from mesenchymal stem cells. Biochem. Biophys. Res. Commun. 491(3), 701-707 (2017).

71. Wicki A, Witzigmann D, Balasubramanian V, Huwyler J. Nanomedicine in cancer therapy: challenges, opportunities, and clinical applications. J. Control. Release 200, 138-157 (2015).

72. Walker S, Busatto S, Pham A et al. Extracellular vesicle-based drug delivery systems for cancer treatment. Theranostics 9(26), 8001-8017 (2019).

73. Tickner JA, Urquhart AJ, Stephenson SA, Richard DJ, O’Byrne KJ. Functions and therapeutic roles of exosomes in cancer. Front. Oncol. 4, 127 (2014).

74. Johnsen KB, Gudbergsson JM, Skov MN, Pilgaard L, Moos T, Duroux M. A comprehensive overview of exosomes as drug delivery vehicles—endogenous nanocarriers for targeted cancer therapy. Biochim. Biophys. Acta 1846(1), 75-87 (2014).

75. Ohno S, Takanashi MF, Sudo KF et al. Systemically injected exosomes targeted to EGFR deliver antitumor microRNA to breast cancer cells. Mol. Ther. 21(1), 185-191 (2013).

76. Bellavia D, Raimondo S, Calabrese G et al. Interleukin 3-receptor targeted exosomes inhibit in vitro and in vivo chronic myelogenous leukemia cell growth. Theranostics 7(5), 1333-1345 (2017).

77. Wang H, Wang B, Zhang A et al. Exosome-mediated miR-29 transfer reduces muscle atrophy and kidney fibrosis in mice. Mol. Ther. 27(3), 571-583 (2019).

78. Limoni SK, Moghadam MF, Moazzeni SM, Gomari H, Salimi F. Engineered exosomes for targeted transfer of siRNA to HER2 positive breast cancer cells. Appl. Biochem. Biotechnol. 187(1), 352-364 (2019).

79. Alvarez-Erviti L, Seow YF, Yin HF, Betts CF, Lakhal S, Wood MJA. Delivery of siRNA to the mouse brain by systemic injection of targeted exosomes. Nat. Biotechnol. 29(4), 341-345 (2011).

80. Bellavia D, Raimondo S, Calabrese G et al. Interleukin 3-receptor targeted exosomes inhibit in vitro and in vivo chronic myelogenous leukemia cell growth. Theranostics 7(5), 1333-1345 (2017).

81. Jia G, Han Y, An Y et al. NRP-1 targeted and cargo-loaded exosomes facilitate simultaneous imaging and therapy of glioma in vitro and in vivo. Biomaterials 178, 302-316 (2018).

82. Nakase I, Futaki S. Combined treatment with a $\mathrm{pH}$-sensitive fusogenic peptide and cationic lipids achieves enhanced cytosolic delivery of exosomes. Sci. Rep. 5, 10112 (2015).

83. Pi F, Binzel DW, Lee TJ et al. Nanoparticle orientation to control RNA loading and ligand display on extracellular vesicles for cancer regression. Nat. Nanotechnol. 13(1), 82-89 (2018).

84. Yuan Z, Kolluri KK, Gowers KH et al. TRAIL delivery by MSC-derived extracellular vesicles is an effective anticancer therapy. J. Extracell. Vesicles 6(1), 1265291 (2017).

85. Ma J, Zhang Y, Tang K et al. Reversing drug resistance of soft tumor-repopulating cells by tumor cell-derived chemotherapeutic microparticles. Cell. Res. 26(6), 713-727 (2016).

86. Hood JL, Scott MJ, Wickline SA. Maximizing exosome colloidal stability following electroporation. Anal. Biochem. 448, 41-49 (2014).

87. Saari H, Lazaro-Ibanez E, Viitala T, Vuorimaa-Laukkanen E, Siljander P, Yliperttula M. Microvesicle- and exosome-mediated drug delivery enhances the cytotoxicity of paclitaxel in autologous prostate cancer cells. J. Control. Release 220(Pt B), 727-737 (2015).

88. Tian Y, Li S, Song J et al. A doxorubicin delivery platform using engineered natural membrane vesicle exosomes for targeted tumor therapy. Biomaterials 35(7), 2383-2390 (2014).

89. Agrawal AK, Aqil F, Jeyabalan J et al. Milk-derived exosomes for oral delivery of paclitaxel. Nanomedicine 13(5), 1627-1636 (2017).

90. Morishita M, Takahashi Y, Matsumoto A, Nishikawa M, Takakura Y. Exosome-based tumor antigens-adjuvant co-delivery utilizing genetically engineered tumor cell-derived exosomes with immunostimulatory CpG DNA. Biomaterials 111, 55-65 (2016).

91. Liang Q, Bie N, Yong T et al. The softness of tumour-cell-derived microparticles regulates their drug-delivery efficiency. Nat. Biomed. Eng. 3(9), 729-740 (2019). 
92. Lee H, Park H, Noh GJ, Lee ES. pH-responsive hyaluronate-anchored extracellular vesicles to promote tumor-targeted drug delivery. Carbohydr. Polym. 202, 323-333 (2018).

93. Wang Y, Chen X, Tian B et al. Nucleolin-targeted extracellular vesicles as a versatile platform for biologics delivery to breast cancer. Theranostics 7(5), 1360-1372 (2017).

94. Kim MS, Haney MJ, Zhao Y et al. Development of exosome-encapsulated paclitaxel to overcome MDR in cancer cells. Nanomedicine 12(3), 655-664 (2016).

95. Pascucci L, Cocce V, Bonomi A et al. Paclitaxel is incorporated by mesenchymal stromal cells and released in exosomes that inhibit in vitro tumor growth: a new approach for drug delivery. J. Control. Release 192, 262-270 (2014).

96. Usman WM, Pham TC, Kwok YY et al. Efficient RNA drug delivery using red blood cell extracellular vesicles. Nat. Commun. 9(1), 2359 (2018).

97. Zhuang X, Teng Y, Samykutty A et al. Grapefruit-derived nanovectors delivering therapeutic miR17 through an intranasal route inhibit brain tumor progression. Mol. Ther. 24(1), 96-105 (2016).

98. Teng Y, Mu J, Hu X, Samykutty A, Zhuang X, Deng Z. Grapefruit-derived nanovectors deliver miR-18a for treatment of liver metastasis of colon cancer by induction of M1 macrophages. Oncotarget 7(18), 25683-25697 (2016).

99. Kammertoens T, Schuler TF, Blankenstein T. Immunotherapy: target the stroma to hit the tumor. Trends Mol. Med. 11(5), 225-231 (2005).

100. Pure E, Lo A. Can targeting stroma pave the way to enhanced antitumor immunity and immunotherapy of solid tumors? Cancer Immunol. Res. 4(4), 269-278 (2016).

101. Kalluri R, Zeisberg M. Fibroblasts in cancer. Nat. Rev. Cancer 6(5), 392-401 (2006).

102. Valenti G, Quinn HM, Heynen GE et al. Cancer stem cells regulate cancer-associated fibroblasts via activation of hedgehog signaling in mammary gland tumors. Cancer Res. 77(8), 2134-2147 (2017).

103. Nagasaki T, Hara M, Nakanishi H, Takahashi H, Sato M, Takeyama H. Interleukin-6 released by colon cancer-associated fibroblasts is critical for tumour angiogenesis: anti-interleukin- 6 receptor antibody suppressed angiogenesis and inhibited tumour-stroma interaction. Br. J. Cancer 110(2), 469-478 (2014).

104. Fukumura D, Xavier RF, Sugiura TF et al. Tumor induction of VEGF promoter activity in stromal cells. Cell 94(6), 715-725 (1998).

105. Erez N, Truitt MF, Olson PF, Arron ST, Hanahan D. Cancer-associated fibroblasts are activated in incipient neoplasia to orchestrate tumor-promoting inflammation in an NF-kB-dependent manner. Cancer Cell 17(2), 135-147 (2010).

106. Sedlakova O, Svastova E, Takacova M, Kopacek J, Pastorek J, Pastorekova S. Carbonic anhydrase IX, a hypoxia-induced catalytic component of the $\mathrm{pH}$ regulating machinery in tumors. Front. Physiol. 4, 400 (2014).

107. Warren CR, Grindel BJ, Francis L, Carson DD, Farach-Carson M. Transcriptional activation by NFkB increases perlecan/HSPG2 expression in the desmoplastic prostate tumor microenvironment. J. Cell. Biochem. 115(7), 1322-1333 (2014).

108. Quinn MJ, Crotty KA, Thompson JF, Coates AS, O’Brien CJ, McCarthy WH. Desmoplastic and desmoplastic neurotropic melanoma: experience with 280 patients. Cancer 83(6), 1128-1135 (1998).

109. Han X, Li Y, Xu Y et al. Reversal of pancreatic desmoplasia by re-educating stellate cells with a tumour microenvironment-activated nanosystem. Nat. Commun. 9(1), 3390 (2018).

110. Breuninger H, Schaumburg-Lever GF, Holzschuh JF, Horny HP. Desmoplastic squamous cell carcinoma of skin and vermilion surface: a highly malignant subtype of skin cancer. Cancer 79(5), 915-919 (1997).

111. Dauer P, Zhao X, Gupta VKe et al. Inactivation of cancer-associated-fibroblasts disrupts oncogenic signaling in pancreatic cancer cells and promotes its regression. Cancer Res. 78(5), 1321-1333 (2018).

112. Hakim N, Patel R, Devoe C, Saif MW. Why HALO 301 failed and implications for treatment of pancreatic cancer. Pancreas (Fairfax) 3(1), e1-e4 (2019).

113. Hu XX, He PP, Qi GB et al. Transformable nanomaterials as an artificial extracellular matrix for inhibiting tumor invasion and metastasis. ACS Nano 11(4), 4086-4096 (2017).

114. Feng B, Zhou F, Hou B et al. Binary cooperative prodrug nanoparticles improve immunotherapy by synergistically modulating immune tumor microenvironment. Adv. Mater. 30(38), e1803001 (2018).

115. Ernsting MJ, Hoang B, Lohse I et al. Targeting of metastasis-promoting tumor-associated fibroblasts and modulation of pancreatic tumor-associated stroma with a carboxymethylcellulose-docetaxel nanoparticle. J. Control. Release 206, 122-130 (2015).

116. Ji T, Ding YF, Zhao YF et al. Peptide assembly integration of fibroblast-targeting and cell-penetration features for enhanced antitumor drug delivery. Adv. Mater. 27(11), 1865-1873 (2015).

117. Zhen Z, Tang W, Wang M et al. Protein nanocage mediated fibroblast-activation protein targeted photoimmunotherapy to enhance cytotoxic T cell infiltration and tumor control. Nano Lett. 17(2), 862-869 (2017).

118. Li L, Zhou S, Lv N et al. Photosensitizer-encapsulated ferritins mediate photodynamic therapy against cancer-associated fibroblasts and improve tumor accumulation of nanoparticles. Mol. Pharm. 15(8), 3595-3599 (2018). 
119. Hu C, Liu X, Ran W et al. Regulating cancer associated fibroblasts with losartan-loaded injectable peptide hydrogel to potentiate chemotherapy in inhibiting growth and lung metastasis of triple negative breast cancer. Biomaterials 144, 60-72 (2017).

120. Baum P, Muller DF, Ruger RF, Kontermann RE. Single-chain Fv immunoliposomes for the targeting of fibroblast activation protein-expressing tumor stromal cells. J. Drug Target. 15(6), 399-406 (2007).

121. Lu JA, Liu XA, Liao YP et al. Breast cancer chemo-immunotherapy through liposomal delivery of an immunogenic cell death stimulus plus interference in the IDO-1 pathway. ACS Nano 12(11), 11041-11061 (2018).

122. Zhang J, Miao L, Guo S et al. Synergistic anti-tumor effects of combined gemcitabine and cisplatin nanoparticles in a stroma-rich bladder carcinoma model. J. Control. Release 182, 90-96 (2014).

123. Hu K, Miao L, Goodwin TJ, Li J, Liu Q, Huang LA. Quercetin remodels the tumor microenvironment to improve the permeation, retention, and antitumor effects of nanoparticles. ACS Nano 11(5), 4916-4925 (2017).

124. Goodwin TJ, Zhou Y, Musetti SN, Liu R, Huang L. Local and transient gene expression primes the liver to resist cancer metastasis. Sci. Transl. Med. 8(364), 364 ra153 (2016).

125. Li X, Qin F, Yang L, Mo L, Li L, Hou L. Sulfatide-containing lipid perfluorooctylbromide nanoparticles as paclitaxel vehicles targeting breast carcinoma. Int. J. Nanomedicine 9, 3971-3985 (2014).

126. Miao L, Li J, Liu Q et al. Transient and local expression of chemokine and immune checkpoint traps to treat pancreatic cancer. ACS Nano 11(9), 8690-8706 (2017).

127. Shen LF, Li JF, Liu QF et al. Local blockade of interleukin 10 and C-X-C motif chemokine ligand 12 with nano-delivery promotes antitumor response in murine cancers. ACS Nano 12(10), 9830-9841 (2018).

128. Miao L, Liu Q, Lin CM et al. Targeting tumor-associated fibroblasts for therapeutic delivery in desmoplastic tumors. Cancer Res. 77(3), 719-731 (2017).

129. Miao L, Wang Y, Lin CM et al. Nanoparticle modulation of the tumor microenvironment enhances therapeutic efficacy of cisplatin. J. Control. Release 217, 27-41 (2015).

130. Lv Y, Xu C, Zhao X et al. Nanoplatform assembled from a CD44-targeted prodrug and smart liposomes for dual targeting of tumor microenvironment and cancer cells. ACS Nano 12(2), 1519-1536 (2018).

131. Hou L, Liu Q, Shen L et al. Nano-delivery of fraxinellone remodels tumor microenvironment and facilitates therapeutic vaccination in desmoplastic melanoma. Theranostics 8(14), 3781-3796 (2018).

132. Ji T, Zhao Y, Ding Y et al. Transformable peptide nanocarriers for expeditious drug release and effective cancer therapy via cancer-associated fibroblast activation. Angew. Chem. Int. Ed. Engl. 55(3), 1050-1055 (2016).

133. Liu Y, Qiao L, Zhang S et al. Dual pH-responsive multifunctional nanoparticles for targeted treatment of breast cancer by combining immunotherapy and chemotherapy. Acta Biomater. 66, 310-324 (2018).

134. Iranpour S, Nejati V, Delirezh N, Biparva P, Shirian S. Enhanced stimulation of anti-breast cancer T cells responses by dendritic cells loaded with poly lactic-co-glycolic acid (PLGA) nanoparticle encapsulated tumor antigens. J. Exp. Clin. Cancer Res. $35(1), 168$ (2016).

135. Zhou H, Fan Z, Deng J et al. Hyaluronidase embedded in nanocarrier PEG shell for enhanced tumor penetration and highly efficient antitumor efficacy. Nano Lett. 16(5), 3268-3277 (2016).

136. Mardhian DF, Storm G, Bansal R, Prakash J. Nano-targeted relaxin impairs fibrosis and tumor growth in pancreatic cancer and improves the efficacy of gemcitabine in vivo. J. Control. Release 290, 1-10 (2018).

137. Xu Z, Ramishetti S, Tseng YC, Guo S, Wang Y, Huang L. Multifunctional nanoparticles co-delivering Trp2 peptide and CpG adjuvant induce potent cytotoxic T-lymphocyte response against melanoma and its lung metastasis. J. Control. Release 172(1), 259-265 (2013).

138. Xu Z, Wang YF, Zhang LF, Huang L. Nanoparticle-delivered transforming growth factor- $\beta$ siRNA enhances vaccination against advanced melanoma by modifying tumor microenvironment. ACS Nano 8(4), 3636-3645 (2014).

139. Stephan MT, Moon JJ, Um SH, Bershteyn A, Irvine DJ. Therapeutic cell engineering with surface-conjugated synthetic nanoparticles. Nat. Med. 16(9), 1035-1041 (2010).

140. Lu Y, Wang Y, Miao L, Haynes M, Xiang G, Huang L. Exploiting in situ antigen generation and immune modulation to enhance chemotherapy response in advanced melanoma: a combination nanomedicine approach. Cancer Lett. 379(1), 32-38 (2016).

141. Huo M, Zhao Y, Satterlee AB, Wang Y, Xu Y, Huang L. Tumor-targeted delivery of sunitinib base enhances vaccine therapy for advanced melanoma by remodeling the tumor microenvironment. J. Control. Release 245, 81-94 (2017). 